\title{
SPECIAL ISSUE EDITORIAL ON COMPUTER-AIDED ADVANCED ANALYTICS
}

\author{
Tieju Ma
}

School of Business, East China University of Science and Technology, Shanghai 200237, China

tjma@ecust.edu.cn $(\bowtie)$

With the rapid development of computer systems, researchers have started conducting advanced analysis on complex systems that previously were very challenging. From the perspective of operational optimization, finding better solutions for large complex systems has always been a challenging issue. From the perspective of complex adaptive systems, agent-based modeling and simulations are viewed as powerful tools to study the emergence of interactions among heterogeneous actors; however, calibrating and validating agent-based models and simulations have been challenging issues. Establishing creative human-involved systems has been attractive in a knowledge-based economy; however, minimal work has been done to evaluate such systems. This special issue presents five research articles that are devoted to the advanced analysis of these challenging issues.

Two articles in this special issue proffer new methods for finding better solutions for large optimization problems. Two articles present the methods and practices of calibrating and validating agent-based modeling and simulations. In addition, another article develops an evaluation method of community service systems from the perspective of knowledge creation, consciousness reform and value co-creation.

\section{Methods for Finding Better Optimal Solutions}

Ruiz and Voß (2016) state that the solvability and quality of solutions that are obtained with a commercial solver is influenced by the hierarchies of mathematical programming formulations with different numbers of variables and constraints, and this becomes very evident if redundant constraints are used. To overcome this obstacle, the researchers propose a related framework for information collection based on these constraints by using the example of a multidimensional multiple-choice knapsack problem (MMKP). In addition, the researchers investigate an application of the framework in maritime shipping and logistics by means of the dynamic berth allocation problem (DBAP), in which optimal solutions are achieved from the root node within the solver.

Zhen et al. (2016) propose a stochastic programming model for the production flow network-oriented supply chain network design problem, which optimizes the decision of allocating stages to suppliers with the objective of minimizing the total expected costs of production and transportation among suppliers under the uncertain demands of customers. Additionally, the researchers develop a local 
branching based solution method to find a solution of the model. The model is applied to a large automobile company; the researchers conduct numerical experiments to validate both the effectiveness of the proposed model and the efficiency of the proposed solution method.

\section{Methods for Calibrating and Validating Agent-Based Models and Simulations}

Shchiptsova et al. (2016) develop a method to calibrate agent-based models with historical observations. In this method, the simulation consistency with historical records is measured as a distance between two vectors of data points, and an inference on parameter values is obtained from the probability distribution of this stochastic estimate. The proposed method is applied to calibrate an agent-based evolution model of the global energy system.

Xia et al. (2016) propose a new agent-based tag-generation model to explain the "staged" power-law distribution of tag usage frequencies, as observed in various social tagging systems. The model is based on a preferential selection mechanism that is influenced by the combinatorial effects of system recommendation and resource multidimensionality. The researchers validate the model by comparing simulation results under different parameter combinations with the distributions of tag usage frequencies in datasets from three famous social tagging systems, Delicious.com, Last.fm and Flickr.

\section{An Approach to the Evaluation of Community Service Systems}

Meng and Nakamori (2016) argue that the value of a community service system must be co-created by collaboration between experts and participants. Based on this argument, the researchers propose an approach to the evaluation of community service systems based on the theory of knowledge construction systems. The approach is applied to evaluate a pioneering community service system in Japan, which provides an education course for elderly men to find their reason for living after retirement.

Tieju Ma is a professor at School of Business, East China University of Science and Technology. His research interest is modeling technological dynamics with uncertainties and heterogeneous agents. 